\title{
A comparison of the effect of a Growing Up Milk - Lite (GUMLi) v. cows' milk on longitudinal dietary patterns and nutrient intakes in children aged 12-23 months: the GUMLi randomised controlled trial
}

\author{
Amy L. Lovell ${ }^{1 *}$, Peter S. W. Davies ${ }^{2}$, Rebecca J. Hill ${ }^{2}$, Tania Milne ${ }^{3}$, Misa Matsuyama ${ }^{2}$, Yannan Jiang ${ }^{4}$, \\ Rachel X. Chen ${ }^{4}$, Cameron C. Grant ${ }^{5,6,7}$ and Clare R. Wall ${ }^{1}$ \\ ${ }^{1}$ Discipline of Nutrition and Dietetics, Faculty of Medical and Health Sciences, The University of Auckland, Private Bag 92019, \\ Auckland 1142, New Zealand \\ ${ }^{2}$ Child Health Research Centre, Level 6 - CHRC Directorate Team, 62 Graham Street, South Brisbane, QLD 4101, Australia \\ ${ }^{3}$ Faculty of Medical and Health Sciences, The University of Auckland, Private Bag 92019, Auckland 1142, New Zealand \\ ${ }^{4}$ Department of Statistics, Faculty of Science, The University of Auckland, Private Bag 92019, Auckland 1142, New Zealand \\ ${ }^{5}$ Department of Paediatrics: Child and Youth Health, Level 12, Support Building, Auckland City Hospital, Park Road, Grafton \\ 1023, New Zealand \\ ${ }^{6}$ Centre for Longitudinal Research, The University of Auckland, School of Population Health, PO Box 18288, Auckland 1743, \\ New Zealand \\ ${ }^{7}$ General Paediatrics, Starship Child Health, Private Bag 92 024, Auckland Mail Centre, Auckland 1142, New Zealand
}

(Submitted 3 August 2018 - Final revision received 24 November 2018 - Accepted 14 December 2018 - First published online 6 February 2019)

\section{Abstract}

The second year of life is a period of nutritional vulnerability. We aimed to investigate the dietary patterns and nutrient intakes from 1 to 2 years of age during the 12-month follow-up period of the Growing Up Milk - Lite (GUMLi) trial. The GUMLi trial was a multi-centre, doubleblinded, randomised controlled trial of 160 healthy 1-year-old children in Auckland, New Zealand and Brisbane, Australia. Dietary intakes were collected at baseline, 3, 6,9 and 12 months post-randomisation, using a validated FFQ. Dietary patterns were identified using principal component analysis of the frequency of food item consumption per $\mathrm{d}$. The effect of the intervention on dietary patterns and intake of eleven nutrients over the duration of the trial were investigated using random effects mixed models. A total of three dietary patterns were identified at baseline: 'junk/snack foods', 'healthy/guideline foods' and 'breast milk/formula'. A significant group difference was observed in 'breast milk/ formula' dietary pattern $z$ scores at 12 months post-randomisation, where those in the GUMLi group loaded more positively on this pattern, suggesting more frequent consumption of breast milk. No difference was seen in the other two dietary patterns. Significant intervention effects were seen on nutrient intake between the GUMLi (intervention) and cows' milk (control) groups, with lower protein and vitamin $\mathrm{B}_{12}$, and higher Fe, vitamin D, vitamin C and Zn intake in the GUMLi (intervention) group. The consumption of GUMLi did not affect dietary patterns, however, GUMLi participants had lower protein intake and higher Fe, vitamins D and C and Zn intake at 2 years of age.

Key words: Dietary patterns: Toddlers: Principal component analysis: Nutrient intake: Growing Up Milk

Nutrition practices established during infancy underpin the development of taste preference, food acceptance, enjoyment of family-style foods and act as a foundation for future eating patterns $^{(1,2)}$. Beyond 1 year of age, once the transition to a mixed diet has been achieved, the focus shifts to increasing the variety of foods offered and allowing children the opportunity to develop their own food preferences ${ }^{(3)}$. Comparative research on the nutrient intake of children 1-2 years of age are limited ${ }^{(4)}$. In a cross-national comparison of four countries (Germany, Russia, USA and Brazil) inadequate intake of $>20 \%$ were seen for vitamins $\mathrm{D}, \mathrm{E}$ and $\mathrm{A}$ and $\mathrm{Ca}^{(4)}$, however, data were not available for all nutrients in each country. In 16-24-month-old children from Australia, inadequate intake $>5 \%$ below the estimated average requirements (EAR) was reported for $\mathrm{Fe}$ $(23 \%)$, vitamin A $(7 \%)$, vitamin C $(14 \%)$ and Ca $(8 \%)^{(5)}$.

The limitations inherent in dietary assessment tools, can result in misinterpretation of nutrient intake data, particularly in young children ${ }^{(6)}$. The assessment of dietary patterns considers the synergistic effects of food that contribute to usual intake, nutrient combinations and habitual consumption ${ }^{(7,8)}$, as it is

Abbreviations: CM, cows' milk; EAR, estimated average requirement; EAT, eating assessment in toddlers; GUM, Growing Up Milk; GUMLi, Growing Up Milk Lite; IQR, interquartile range; PCA, principal component analysis; RCT, randomised controlled trial.

* Corresponding author: A. L. Lovell, fax +6493035962, email a.lovell@auckland.ac.nz 
likely there are additive effects of food constituents on health. Principal component analysis (PCA) is a multivariate, $a$ posteriori, data-driven reduction technique commonly used to summarise dietary data ${ }^{(9,10)}$; and in a recent systematic review, FFQ were found to be the most common method used to derive dietary patterns in children under the age of 5 years ${ }^{(11)}$. Few studies have described the dietary patterns of children under 2 years $^{(7,12-16)}$ or the energy and nutrient intake during the dietary transition from complementary to family foods ${ }^{(16-18)}$.

Growing Up Milk (GUM) or young child formula are a milkbased alternative to cows' milk (CM) or breast milk for children 1-3 years of age, fortified with nutrients that are commonly low during the transition to family-based foods ${ }^{(19)}$. Although not necessary for adequate nutrition, GUM may compensate for nutritional deficiencies during this period through improving nutritional status ${ }^{(20)}$, but little is known about their effects on dietary patterns and nutrient intake over the entire second year of life. There are no reported safety issues associated with the use of GUM, however, concerns remain over its use to provide adequate nutrition in place of a varied diet, increasing dependence on a 'liquid dietary intake' over regular food consumption and disrupting satiety ${ }^{(19,21,22)}$. In a recent position statement, the European Society for Paediatric Gastroenterology, Hepatology and Nutrition (ESPGHAN) conclude that there is no necessity for routine use of GUM but suggest they may have a small role to play as a strategy to increase the intake of nutrients such as $\mathrm{Fe}$ and vitamin $\mathrm{D}$ during this $\operatorname{period}^{(19)}$.

No studies have described the dietary patterns in New Zealand children aged 1-2 years, and data on Australian children remain limited $^{(23)}$. We aimed to evaluate the prospective wholeof-diet patterns in the children enrolled in the Growing Up Milk - Lite (GUMLi) trial using PCA, assess their energy and nutrient intakes and assess the impact of the GUMLi intervention over the 12 -month trial period.

\section{Methods}

\section{Study design}

The GUMLi trial was a multicentre, two-arm, double-blinded, randomised controlled trial (RCT) consisting of 160 healthy children of 1-year of age, living in Auckland ( $n$ 108) and Brisbane ( $n$ 52). The main trial evaluated the effect of consuming GUMLi (reduced protein) compared with unfortified CM as part of a whole diet for 12 months, on body composition at 2 years of age (primary outcome) ${ }^{(24)}$.

Secondary outcomes included measures of dietary intake; anthropometry; micronutrient status and cognitive development. The study was conducted according to the guidelines laid down in the Declaration of Helsinki and ethical approval was obtained from the Northern B Health and Disability Ethics Committee, Ministry of Health, New Zealand (14/ NTB/152) and the University of Queensland Medical Research Ethics Committee, Brisbane, Australia (2014001318). Written, informed consent was provided by the primary caregivers.

\section{Study intervention}

Participants were randomised 1:1 to GUMLi (intervention) or CM (control) ( $n 80$ per group) for a period of 12 months. The study milks were provided at no cost to participants and were produced in powder form, packaged in plain, identical 900-g tins, with no additional nutrient information panels or nutritionrelated statements. The study milks were independently allocated by Danone Nutricia Research. Both researchers and participants were blinded to treatment allocation until completion of the trial. Parents were not given any dietary advice during the intervention and breast-feeding continued throughout the trial, if desired. Any use of other infant formulas at baseline was discontinued after randomisation.

The compositional differences between the two study milks are described in Table 1. GUMLi had a reduced energy and protein content when compared with standard, commercial GUM on the market, $249 \mathrm{~kJ} / 100 \mathrm{ml} v .298 \mathrm{~kJ} / 100 \mathrm{ml}$ and $1.7 \mathrm{~g} /$ $100 \mathrm{ml}$ protein $v \cdot 2 \cdot 2 \mathrm{~g} / 100 \mathrm{ml}$. An energy-matched, non-fortified $\mathrm{CM}$ was used as an active control, with a protein content of $3 \cdot 1 \mathrm{~g} / 100 \mathrm{ml}$. In addition, GUMLi was fortified with Fe and vitamin D. As with commercially available GUM, GUMLi was fortified with vitamin $\mathrm{C}$ to increase the bioavailability of nonheme Fe. The amount of vitamin C in GUMLi was higher than the standard GUM $(17 \mathrm{mg} / 100 \mathrm{ml} v$. approximately $15 \mathrm{mg} /$ $100 \mathrm{ml})$.

\section{Child dietary assessment}

Diet was assessed via a face-to-face interview, using the previously validated, interviewer-administered Eating Assessment in Toddlers (EAT) FFQ at baseline, 3, 6, 9 and 12 months postrandomisation. The EAT FFQ is designed to describe dietary intake over the previous 4 weeks, with good validity and high

Table 1. Nutritional composition of cows' milk (CM) and Growing Up Milk - Lite (GUMLi) per $100 \mathrm{ml}$ of prepared product

\begin{tabular}{|c|c|c|}
\hline & \multicolumn{2}{|c|}{ Study group } \\
\hline & Control $\mathrm{CM}^{*}$ & $\begin{array}{l}\text { Intervention } \\
\text { GUML }^{*}\end{array}$ \\
\hline Energy (kJ) & $245 \cdot 0$ & $249 \cdot 0$ \\
\hline Energy (kcal) & 71.0 & $60 \cdot 0$ \\
\hline \multicolumn{3}{|l|}{ Macronutrients } \\
\hline Protein $(\mathrm{g})$ & 3.1 & 1.7 \\
\hline Carbohydrate (g) & 4.5 & $7 \cdot 8$ \\
\hline Total fat (g) & $3 \cdot 1$ & 1.9 \\
\hline Saturated (g) & 1.9 & 1.3 \\
\hline $\begin{array}{l}\text { Total } n-3 \text { long-chain fatty acids (g) } \\
(\mathrm{DHA}+\mathrm{EPA}+\mathrm{DPA})\end{array}$ & $<0.002$ & 0.04 \\
\hline \multicolumn{3}{|l|}{ Dietary fibre } \\
\hline scGOS (g) & 0.0 & 1.8 \\
\hline IcFOS $(\mathrm{g})$ & 0.0 & 0.2 \\
\hline \multicolumn{3}{|l|}{ Micronutrients } \\
\hline Non-haeme Fe (mg) & 0.0 & $1 \cdot 3$ \\
\hline Cholecalciferol $(\mu \mathrm{q})$ & 0.1 & 1.2 \\
\hline Vitamin $B_{12}(\mu \mathrm{g})$ & 0.4 & 0.14 \\
\hline Vitamin C (mg) & - & $17 \cdot 0$ \\
\hline
\end{tabular}

DPA, docosapentaenoic acid; scGOS, short-chain galacto-oligosaccharides; IcFOS, long-chain fructo-oligosaccharides.

* Obtained from manufacturer, values based on average totals from three batches produced for use in the GUMLi trial. 
reproducibility for intake of macronutrients, key micronutrients ${ }^{(25)}$ and dietary patterns ${ }^{(26)}$. The EAT FFQ contained ninety-nine food items that were collapsed into sixteen nonoverlapping food groups based on nutrient profile and similarity of use, as previously described ${ }^{(25,26)}$. Further information about the sixteen food groups and food items assigned to each group can be found in online Supplementary material (online Supplementary Table S1). A total of eleven nutrients were derived using the original EAT FFQ design and included nutrients such as Fe and vitamin D, identified by the European Food Safety Authority as key nutrients that may require specific attention in children $1-3$ years of age ${ }^{(27)}$.

\section{Nutrient intake}

The amount (g) consumed per d for each food item was calculated by multiplying the frequency of consumption per $\mathrm{d}$, volume (g) and the total amount eaten. Volume was calculated as natural food portions or using the child's palm volume ${ }^{(25,26,28)}$. Nutrients were calculated as previously described ${ }^{(25)}$. Nutrient analyses were performed in MATLAB ${ }^{\circledast}$ (MathWorks Inc.), using a custom written programme verified by hand calculations. Implausible energy intakes were identified using Tukey's fences method of interquartile range (IQR) calculated as a lower cut-off of (Q1$(1.5 \times \mathrm{IQR}))$ and upper cut-off of $(\mathrm{Q} 3+(1.5 \times \mathrm{IQR}))^{(29)}$ and full dietary data excluded from the analysis ${ }^{(29)}$. Before identifying the baseline dietary patterns, three participants were excluded due to implausible energy intakes ( $n$ 157).

\section{Identifying dietary patterns}

Dietary patterns were derived using exploratory PCA of the baseline EAT FFQ administration ( $n$ 157). The frequency of intake per $\mathrm{d}$ for the sixteen food groups was used. The use of frequency of intake data allowed the inclusion of breast milk, as the portion size could not be estimated. The number of components was identified using the breakpoint on the scree plot $^{(30)}$, eigenvalues $>1$, components that account for at least $10 \%$ of the total variance and component interpretability after varimax rotation. Varimax (orthogonal) rotation was performed to maximise the variance within components, resulting in uncorrelated factors ${ }^{(31)}$. Food groups with an absolute factor loading $\geq 0.3$ on a component were considered to have a strong association. The highest absolute loading score for each food group was considered the most informative in describing the dietary pattern. For each pattern identified at baseline, an individual score for each participant was created at each visit by multiplying the factor loadings by the corresponding standardised value for each food group and summing across the food groups. A positive factor loading score was associated with a higher standardised $z$ score per food group consumption for an individual participant, and a negative factor loading score indicated an inverse association.

\section{Statistical analysis}

Statistical analyses were performed using SAS version 9.4 (SAS Institute Inc.), following the principle of intention to treat, including all randomised participants who provided valid trial data for analysis. All statistical tests were two sided at a $5 \%$ level of significance. Participant demographics and baseline characteristics were summarised by treatment group using descriptive statistics. Categorical variables were described as frequencies and percentages; continuous variables as means and standard deviations.

Dietary intakes for the sixteen food groups assessed by the EAT FFQ were summarised by intervention group at each visit. Dietary patterns were identified using PCA on the baseline dietary data. An individually standardised $z$ score was calculated for each participant and dietary pattern at baseline and converted into quartiles. The differences in eleven key nutrients between participants in different quartiles were tested using the Kruskal-Wallis test.

Adherence to the dietary patterns identified at baseline was measured over time, using the scoring coefficients from baseline applied to each child's food intake at later follow-up time points ${ }^{(32,33)}$. The impact of the GUMLi intervention on the dietary pattern individual $z$ scores and average daily nutrient intake compared with CM was evaluated at each time point postrandomisation, using random effects mixed models with an interaction term between treatment group and time point, adjusting for baseline outcome value and study location. An unstructured correlation structure was applied to repeated measures on the same participant over time with a random cluster effect. Missing data at follow-up visits were taken into account in the model using the maximum likelihood method, assuming they were missing at random. Model-adjusted mean differences and $95 \%$ CI were reported at each time point with associated $P$ values.

\section{Results}

\section{Population baseline characteristics}

A total of 160 eligible children aged 1 year were randomly assigned 1:1 to receive the intervention (GUMLi) or nonfortified $\mathrm{CM}$ in the main trial. Characteristics of the two treatment groups included in the analysis of patterns and nutrients at 1 year of age are reported in Table 2 ( $n$ 157). A total of three participants (one GUMLi and two CM) were excluded from the analysis of dietary patterns and nutrients due to implausible estimated energy intake using the method described by Huang et $a l .{ }^{(29)}$ Tukey's fences method of IQR were calculated as a lower cut-off (Q1 - (1.5 × IQR)) and upper cut-off (Q3 + (1.5× IQR)). A flowchart of participants with complete dietary data and plausible estimated energy intake during the study period is reported in online Supplementary material (online Supplementary Fig. S1). There were no differences between treatment groups at baseline. Approximately half the participants were the only child, $40 \%$ were breastfed at baseline and mothers were predominantly European and had achieved secondary education or higher.

\section{Sixteen food groups}

Mean (SD) daily intakes of the sixteen food groups expressed as frequency per $\mathrm{d}$ at each time point are displayed in online 
Table 2. Characteristics of the cohort included in the analysis of patterns and nutrients at 1 year of age (Numbers and percentages)

\begin{tabular}{|c|c|c|c|c|}
\hline \multirow[b]{3}{*}{ Baseline demographics } & \multicolumn{4}{|c|}{ Study group } \\
\hline & \multicolumn{2}{|c|}{ Intervention ( $n$ 80) } & \multicolumn{2}{|c|}{ Control ( $n 77)$} \\
\hline & $n$ & $\%$ & $n$ & $\%$ \\
\hline \multicolumn{5}{|l|}{ Location } \\
\hline Auckland & 52 & 65 & 55 & 71 \\
\hline Brisbane & 28 & 35 & 22 & 29 \\
\hline \multicolumn{5}{|l|}{ Gestation (completed weeks gestation) } \\
\hline Term ( $\geq 37$ weeks) & 78 & 98 & 70 & 91 \\
\hline Pre-term ( $<37$ weeks) & 2 & 2 & 7 & 9 \\
\hline \multicolumn{5}{|l|}{ Child's sex } \\
\hline Boy & 40 & 50 & 44 & 57 \\
\hline Girl & 40 & 50 & 33 & 43 \\
\hline \multicolumn{5}{|l|}{ Other children in the family } \\
\hline No & 40 & 50 & 36 & 47 \\
\hline Yes & 40 & 50 & 41 & 53 \\
\hline \multicolumn{5}{|l|}{ Breastfed at baseline } \\
\hline No & 47 & 60 & 45 & 58 \\
\hline Yes & 31 & 40 & 32 & 42 \\
\hline Missing & 2 & & & \\
\hline \multicolumn{5}{|l|}{ Mother's ethnicity } \\
\hline Māori & 8 & 10 & 7 & 9 \\
\hline Pacific & 0 & 0 & 3 & 4 \\
\hline Asian & 3 & 4 & 5 & 6 \\
\hline European & 56 & 70 & 49 & 64 \\
\hline Other & 13 & 16 & 13 & 17 \\
\hline \multicolumn{5}{|l|}{ Mother's highest level of education } \\
\hline No school qualifications & 0 & 0 & 0 & 0 \\
\hline Primary & 2 & 3 & 0 & 0 \\
\hline Secondary & 12 & 15 & 14 & 18 \\
\hline Tertiary & 62 & 78 & 56 & 73 \\
\hline Other & 4 & 5 & 7 & 9 \\
\hline \multicolumn{5}{|l|}{ Mother's employment status } \\
\hline Full-time caregiver & 23 & 29 & 22 & 29 \\
\hline Full-time paid employment & 16 & 20 & 22 & 29 \\
\hline Part-time paid employment & 28 & 35 & 29 & 38 \\
\hline Receiving a benefit & 2 & 3 & 1 & 1 \\
\hline Unemployed, not receiving a benefit & 4 & 5 & 1 & 1 \\
\hline Other & 7 & 9 & 1 & 1 \\
\hline Prefer not to answer & 0 & 0 & 1 & 1 \\
\hline \multicolumn{5}{|l|}{ Current partner } \\
\hline No & 1 & 1 & 2 & 3 \\
\hline Yes & 79 & 99 & 74 & 96 \\
\hline Prefer not to answer & 0 & 0 & 1 & 1 \\
\hline \multicolumn{5}{|l|}{ Smoking } \\
\hline Current smoking & 2 & 3 & 2 & 3 \\
\hline Smoking before pregnancy & 7 & 9 & 6 & 8 \\
\hline Smoking during pregnancy & 1 & 1 & 1 & 1 \\
\hline
\end{tabular}

Supplementary Table S2. Over time, there were no differences in the frequency of intake between GUMLi or CM groups for baby/toddler food; bread/pasta/low-sugar-cereal; meat; eggs/beans; fruit; milk and milk products; breast milk; hot chips/roast potato/kumara and savoury snacks. There were no differences in the frequency of intake of toddler milk and infant formula, as from month 3 of the intervention this category recorded consumption of the study milk, that is, $300 \mathrm{ml} / \mathrm{d}$ of group A (CM) and group B (GUMLi). Children in the GUMLi group had a higher mean frequency of intake of processed meats at month 6 , and consistently higher frequency of intake of vegetables following randomisation (i.e. from month 3). However, the GUMLi group also had a higher frequency of intake of sweet foods at baseline and month 9 and the CM group had a higher frequency of intake of sweet drinks at month 6 and spreads and savoury snacks at month 3 .

\section{Baseline dietary patterns}

A total of three major dietary patterns were identified using PCA at baseline, accounting for $39 \%$ of the variability within the sample, with the first pattern explaining $15 \%$, followed by 13 and $11 \%$ in the second and third patterns, respectively. The first dietary pattern 'junk/snack foods' was characterised by positive loadings on hot chips/roast potato/kumara, processed meats, sweet foods, nutritive drinks, savoury snacks, sweet drinks and spreads (by descending order of PCA 
Table 3. Food groups included in principal component analysis and varimax-rotated factor loading of the three dietary patterns at baseline

\begin{tabular}{|c|c|c|c|}
\hline \multirow[b]{2}{*}{ Sixteen food group daily intake } & \multicolumn{3}{|c|}{ Dietary pattern } \\
\hline & Junk/snack foods & Healthy/guideline foods & Breast milk/formula \\
\hline Baby/toddler food & $0 \cdot 18$ & $-0.56^{\star}$ & 0.03 \\
\hline Bread, pasta & 0.21 & $0.52^{*}$ & -0.05 \\
\hline Meat, fish & -0.12 & $0.53^{*}$ & 0.08 \\
\hline Processed meat & $0.66^{*}$ & 0.15 & -0.22 \\
\hline Eggs, beans & 0.02 & $0.39^{\star}$ & -0.07 \\
\hline Vegetables & $-0 \cdot 15$ & $0.71^{*}$ & 0.01 \\
\hline Fruit & -0.24 & $0.55^{\star}$ & 0.19 \\
\hline Milk, milk products & 0.13 & $0.40^{*}$ & 0.03 \\
\hline Breast milk & -0.06 & -0.14 & $0.84^{*}$ \\
\hline Toddler milk and infant formula & -0.04 & -0.20 & $-0.83^{\star}$ \\
\hline Spreads (margarines, butter) & $0 \cdot 35^{\star}$ & $0 \cdot 35^{\star}$ & $-0 \cdot 14$ \\
\hline Cakes & $0.60^{*}$ & -0.01 & $0.33^{*}$ \\
\hline Sweet drinks & $0.41^{*}$ & -0.04 & -0.09 \\
\hline Hot chips & $0.68^{*}$ & -0.08 & 0.23 \\
\hline Savoury snacks & $0.51^{\star}$ & -0.01 & -0.05 \\
\hline Nutritive drinks & $0.53^{*}$ & -0.25 & 0.05 \\
\hline
\end{tabular}

${ }^{*}$ Loading $\geq 0.30$ to aid labelling of dietary patterns.

loadings), and with negative loadings on formula, breast milk, meat/fish, vegetables and fruit. The 'healthy/guideline foods' dietary pattern was positively associated with vegetables, fruit, bread/pasta, meat/fish, milk/milk products, eggs/beans and spreads, with negative loadings on sweet foods, savoury snacks, sweet drinks, fries/roast potato/kumara, breast milk, formula, nutritive drinks and baby/toddler food. The "breast milk/formula' dietary pattern loaded with opposite strength on breast milk (0.84) and formula (including GUM) (-0.82) (Table 3).

\section{Construct validity of dietary patterns}

Baseline nutrient intakes according to quartiles of dietary pattern scores were identified and quartiles 1 (Q1) and 4 (Q4) are displayed in online Supplementary Table S3. Those in Q1 had individual scores that were least associated with the dietary pattern, and those in Q4 had scores most associated with the dietary pattern. Higher scores on the junk/snack food' pattern were associated with higher total energy, protein, fat and carbohydrate intake, and higher scores on the 'healthy/ guideline foods' pattern were associated with higher total energy, protein, total fat, fibre, $\mathrm{Zn}$ and vitamin $\mathrm{B}_{12}$ intake. Both the 'junk/snack food' pattern and the 'healthy/guideline foods' pattern showed significant trends for total energy, protein and total fat intakes, increasing from Q1 to Q4. To elucidate any differences in these trends, the difference in nutrient intake between Q4 and Q1 was calculated. Scores on the 'junk/snack' pattern and 'healthy/guideline' pattern were associated with $1143.9 v .825 .9 \mathrm{~kJ}$ difference in median energy, a $17.1 v .19 \cdot 2 \mathrm{~g}$ difference in median protein and $12.7 v .15 .2 \mathrm{~g}$ difference in median total fat intake between Q4 and Q1, respectively. Higher scores on the 'breast milk/formula' pattern were associated with lower intake of total energy, protein, fat, carbohydrate, $\mathrm{Ca}, \mathrm{Fe}, \mathrm{Zn}$ and vitamins $\mathrm{B}_{12}$, C and D compared with those in $\mathrm{Q} 1$ who were less adherent to the dietary pattern, thereby consuming more formula.

\section{Effect of the intervention on dietary patterns over time}

The effect of the intervention on dietary patterns at each time point was investigated. Standardised $z$ scores of the dietary patterns at baseline, 3, 6, 9 and 12 months post-randomisation are presented in Table 4. No significant differences in the $z$ scores were seen between CM and GUMLi groups for the 'junk/snack foods' or 'healthy/guideline foods' patterns over time. A significant group difference was observed in the 'breast milk/formula' dietary pattern $z$ scores at 12 months postrandomisation $(P=0.041)$, where those in the GUMLi group had more a positive loading on the dietary pattern.

\section{Nutrient intake}

Mean (SD) nutrient intakes/d at baseline, 3, 6, 9 and 12 months post-randomisation are displayed in online Supplementary Table S4. There was no difference in the total intake of carbohydrates, fibre and $\mathrm{Ca}$ consumed per $\mathrm{d}$ between groups. At month 3, the GUMLi group had a lower total energy intake compared with the CM group $(P=0.012)$. Total protein intake was significantly different at months 3 and $9(P<0.0001$ and $P=0.0042$, respectively), where the $\mathrm{CM}$ group consumed higher total protein intake. By 2 years of age, the GUMLi group consumed $65.8 \mathrm{~g} / \mathrm{d}$ protein compared with $69.0 \mathrm{~g} / \mathrm{d}$ in the CM group $(P=0.451)$. The contribution of protein according to the sixteen EAT FFQ food groups was calculated (data not shown). Both groups had similar protein intake from milk and milk products (19.2 v. $19.9 \mathrm{~g} / \mathrm{d}, \mathrm{CM}$ and GUMLi, respectively), and the impact of a lower protein intervention milk was apparent $(9.8 v .4 .9 \mathrm{~g} / \mathrm{d}, \mathrm{CM}$ and GUMLi, respectively). Total fat intake per $\mathrm{d}$ was similar between groups, except for month 3 , where the CM group consumed a greater amount $(P<0 \cdot 0001)$. There was a significant difference in total $\mathrm{Fe}$ consumption between $\mathrm{CM}$ and GUMLi groups, with the GUMLi group consuming higher total Fe intake per $\mathrm{d}$ from month $3(P<0 \cdot 0001)$. No children exceeded the upper limit for $\mathrm{Fe}$ of $21.0 \mathrm{mg} / \mathrm{d}^{(34)}$. At month 6 , the GUMLi group had a greater total $\mathrm{Zn}$ intake $(P=0.0202)$. 
From month 3, those in the GUMLi group consumed significantly more vitamins $\mathrm{C}$ and $\mathrm{D}$ compared with the CM group $(P<0.0001)$. From month 3 , the $\mathrm{CM}$ group had significantly higher total vitamin $\mathrm{B}_{12}$ intake compared with the GUMLi group.

The EAR cut point method ${ }^{(35,36)}$ was used to determine the number of participants that did not meet the nutrient reference values $^{(34)}$ for the eleven nutrients specified by the EAT FFQ (data not shown). After 12 months of the intervention, 86\% ( $n$ 61) of the CM participants and 9\% $(n 6)$ of the GUMLi participants had intake less than the EAR for vitamin C (GUMLi: $60.3 \mathrm{mg} / \mathrm{d}$; CM: $19.3 \mathrm{mg} / \mathrm{d}$; EAR: $25.0 \mathrm{mg} / \mathrm{d} ; P<0.0001)$. In all, $24 \%(n 17)$ of the CM participants and $1.5 \%(n 1)$ for the GUMLi participants did not meet the EAR for Fe (GUMLi: $10 \cdot 2 \mathrm{~g} / \mathrm{d}$; CM: $5.8 \mathrm{~g} / \mathrm{d}$; EAR: $4.0 \mathrm{~g} / \mathrm{d} ; P<0.0001)$ and $23 \%(n 16)$ of the CM participants and $9 \%(n 6)$ of the GUMLi participants did not meet the adequate intake (AI) for vitamin D (GUMLi: $7 \cdot 2 \mu \mathrm{g} / \mathrm{d}$; CM: $4.4 \mu \mathrm{g} / \mathrm{d} ; \mathrm{AI}: 5 \mu \mathrm{g} / \mathrm{d} ; P<0.0001)$

\section{Discussion}

In this RCT, we evaluated the effect of consuming a reduced protein, micronutrient-fortified GUM (GUMLi) compared with unfortified CM for 12 months on dietary patterns. This is the first study to use an applied dietary pattern $z$ score on longitudinal trial data. Our findings suggest that consumption of GUMLi did not affect dietary patterns, however, lowered protein intakes and improved intake of $\mathrm{Fe}$, vitamins $\mathrm{D}$ and $\mathrm{C}$ and $\mathrm{Zn}$ when compared with CM. We have also been able to identify trends in nutrient intake across dietary pattern scores identified at baseline, demonstrating that the EAT FFQ is able to describe dietary patterns and differences in both food and nutrient intake in children under 2 years of age.

\section{Identified dietary patterns}

A total of three clear patterns were identified at baseline and demonstrated similarities with cohorts of children at similar ages, including the Avon Longitudinal Study of Parents and Children (ALSPAC) ${ }^{(12)}$, the Southampton Women's Study $(\mathrm{SWS})^{(7)}$, the Norwegian Mother and Child Cohort $(\mathrm{MoBa})^{(37)}$ and the French Etude des Déterminants du développement et de la santé de l'ENfant (EDEN) cohort ${ }^{(38)}$. Our first pattern, 'junk/snack food' identified foods commonly found in an adult snack-type dietary intake, as seen in the ALSPAC 'biscuits, sweets and crisps' pattern ${ }^{(12)}$, the SWS 'adult foods' pattern ${ }^{(7)}$, the MoBa 'unhealthy' pattern ${ }^{(37)}$ and the EDEN 'processed and fast foods' pattern ${ }^{(38)}$. Our second pattern, 'healthy/guideline foods' was similar to the ALSPAC 'meat, vegetables and desserts' pattern $^{(12)}$, the SWS 'infant guidelines' pattern ${ }^{(7)}$, the MoBa 'wholesome' pattern ${ }^{(37)}$ and the EDEN 'guidelines' pattern ${ }^{(38)}$ and was influenced by foods seen to conform to infant feeding guidelines. In New Zealand, dietary patterns have been described in older paediatric populations, where 'junk', 'healthy' and 'traditional' patterns were identified at 3.5 and 7 years of age ${ }^{(39)}$, and in 14- and 24-month-old Australian children, patterns that reflected core and non-core food intake were identified $^{(23)}$. Across both studies, the identified patterns 
consisted of similar food items to our 'junk/snack foods' and 'healthy/guideline foods' patterns.

Our third pattern, 'breast milk/formula' loaded with opposite strength on breast milk (0.84) and formula (including GUM) $(-0 \cdot 82)$, where the negative direction of the association indicates that lower scores are associated with a higher consumption of formula. Over time, the loadings were negatively associated with the pattern, indicating that formula consumption was increasing, but those in the GUMLi group were consuming more breast milk at these ages compared with the CM group. A similar relationship was identified in the 'breast-feeding' pattern in the 6-month-old infants from the ALSPAC study, where a high positive loading on breast milk (0.78) and high negative loading on infant formula $(-0.76)$ were seen ${ }^{(17)}$. Although identified in early infancy in the UK, children in our study still loaded on our 'breast milk/formula' pattern at months 9 and 12 of the intervention, when the children were 21 and 24 months of age, respectively. The relationship between breast-feeding and GUM intake has also been identified in the NutriBébé Survey in France, where a higher percentage of mothers still breastfeeding or who previously breastfed gave GUM to their children $(32 \%)$ compared with mothers that did not $(25 \%)^{(40)}$

\section{Nutrient intakes}

To date, only two studies described the construct validity of dietary patterns in terms of the underlying nutrient profiles in children under 2 years of age, confirming that dietary patterns are able to measure the differences in combinations of nutrient densities ${ }^{(17,23)}$. Using a similar approach, we were able to demonstrate trends in the underlying nutrient intake across quartiles of baseline dietary pattern scores. It is important to note that this analysis was performed on baseline data, before allocation into treatment group and cessation of any additional infant, follow-on formula or GUM, which would have contributed to both macro- and micronutrient intake.

The differences between 'junk/snack' and 'healthy/guideline' dietary patterns were difficult to interpret. Both patterns were positively associated with total energy, protein and total fat; however, in addition, the 'healthy/guideline' pattern was positively associated with fibre, $\mathrm{Zn}$ and vitamin $\mathrm{B}_{12}$, reflecting a more positive nutrient profile, with scope for further improvement. The EAT FFQ did not differentiate between types of fats, that is, saturated $v$. polyunsaturated, which may have contributed to both patterns having a positive association with total fat intake due to all fats contributing the same amount of energy $(37.7 \mathrm{~kJ})$ per g. In contrast, the highest quartile of the breast milk/formula' pattern had a negative association with total energy, protein, fat, carbohydrate, $\mathrm{Ca}, \mathrm{Fe}, \mathrm{Zn}$ and vitamins $\mathrm{B}_{12}$, $\mathrm{C}$ and D. Frequency (per d) of breast-feeding was used in the baseline PCA, however, volume and nutrient content estimates were not directly assessed. As described in the literature from this age group, the decision was made not to exclude participants that were breast-feeding as a large portion of the dietary data would be missed, reducing the ability of the FFQ to describe the total diet. Collecting accurate data on the amount of breast milk consumed and its nutrient content would have required breast milk collection and test weighing, both of which were not feasible in this study, as the data collection burden was significant for participants, with further measures increasing the likelihood of participant attrition and loss of statistical power. Therefore, associations between scores on the breast milk/formula' pattern and underlying nutrients may be less reliable but may still reflect an age-appropriate nutrient profile, also reported by Smithers et al. ${ }^{(17)}$ in an analysis of the relationship with nutrient intake at 8 and 15 months of age and dietary patterns at 6 and 15 months of age.

\section{Effect of the intervention over time}

The effect of GUM on energy and nutrient intakes has been assessed to a limited capacity in six cross-sectional studies ${ }^{(41-46)}$ and five RCT study designs ${ }^{(47-51)}$, all performed in developed countries. Most studies focused on single-nutrient intake (i.e. Fe or vitamin D) ${ }^{(46,49-51)}$, with only one other $\mathrm{RCT}^{(47)}$ describing multiple-nutrient intakes according to CM or GUM, presented as a supplementary analysis. The compositional differences between the two intervention milks (GUMLi $v$. CM) resulted in significant differences in protein and key micronutrients (i.e. Fe and vitamin D) between groups. After 12 months, the children drinking GUMLi had a daily Fe intake $2 \cdot 7$ times the current $\mathrm{EAR}^{(52)}$. These increases were similar to those seen in another New Zealand study, where children in an Fe-fortified milk group had daily $\mathrm{Fe}$ intake 2.5 times the EAR compared with an unfortified control milk or red meat group after 20 weeks ${ }^{(48)}$. Consumption of GUMLi did not lead to excessive intake of nutrients compared with $\mathrm{CM}$ (online Supplementary Table S4). In addition to Fe, GUMLi participants were more likely to have vitamins $\mathrm{C}$ and $\mathrm{D}$ intake that were greater than the $\mathrm{EAR}$ and $\mathrm{AI}$, respectively; however, no participants exceeded the upper limits for these nutrients ${ }^{(34)}$.

Greater mean protein intake compared with the literature was observed, with the CM group consuming larger quantities of protein at all time points and significant differences in intake at months 3 and 9. In a study of 12-24-month-old French children, those drinking CM had mean protein intake of $42 \mathrm{~g} / \mathrm{d}$ and children drinking GUM had protein intake of $36 \mathrm{~g} / \mathrm{d}$ $(P<0.0001)^{(41)}$. Supplementary analyses from a 12-month RCT in 1-4-year-old children in India ${ }^{(47)}$ described no differences in energy intake between children consuming a micronutrient fortified milk and a non-fortified control milk. No differences were seen in protein intake after 6 months of the intervention, but significant differences were seen after 12 months $(P=0 \cdot 01)$. However, as both milks had similar protein contents, this difference cannot solely be attributed to differences in milk intake. In our study, by 2 years of age, the children in the GUMLi group obtained larger amounts of protein from other food groups, that is, fruits, vegetables and meat/fish. Further research is indicated, but this may imply that the children in the GUMLi group experienced an earlier transition from a milk-based intake to an intake more reflective of family-based foods, with greater variety compared with the CM group.

\section{Strengths and limitations}

Strengths of our study include a data collection period of 52 weeks, spanning half the 'at-risk' toddler period of 1-3 years 
of age, and twice the time frame of studies with similar outcomes ${ }^{(48,53)}$ and the use of a validate $\mathrm{FFQ}^{(25)}$. To improve the accuracy of the use of self-reported data, three adjustments were made to the EAT FFQ before its validation. Cross-check questions were added to the fruits and vegetables question, in an attempt to calculate an adjustment factor for each consumption frequency within the group, a technique that has been shown to reduce overestimation when long itemised lists are included in $\mathrm{FFQ}^{(54)}$. In an effort to reduce the effect of portion size estimation leading to overestimation of intake, a unique methodology was adopted for the EAT FFQ, where the participants palm size was used as a reference and questions accounted for leftovers through asking for portion size information of the average amount offered each time and the amount actually eaten ${ }^{(25)}$. Our novel approach of calculating applied dietary pattern $z$ scores for five-time points using factor loadings from the baseline dietary patterns has allowed assessment of change in baseline dietary patterns over time and evaluation of the impact of the intervention. However, this methodology does not allow detection of any new dietary patterns that may arise at later time points ${ }^{(55)}$. Limitations of the study include the use of PCA which can be influenced by the way in which foods are grouped ${ }^{(56)}$ and, as PCA is a data-driven method, results from this analysis cannot be extrapolated to other populations. The EAT FFQ included a complex method of reporting portion size where the child's palm volume was used. This may have resulted in miss reporting portion sizes. As the intervention progressed, parents became more familiar with this method. n-3 PUFA (including DHA, EPA and docosapentaenoic acid (DPA)) were not included in the nutrient lines of the EAT FFQ. Although identified by the European Food Safety Authority $^{(57)}$ and Food and Agriculture Organization/World Health Organization $^{(58)}$ as a nutrient whose intake are below the recommended levels in 1-3-year-old children, we were not able to determine the estimated intake from food in our population. GUMLi was fortified with a total n-3 PUFA (including DHA, EPA, DPA) of $40 \mathrm{mg} / 100 \mathrm{ml}$. Therefore, consumption of the prescribed $300 \mathrm{ml} / \mathrm{d}$ would result in $120 \mathrm{mg}$ of $n-3$ PUFA/d (DHA, EPA and DPA) in the GUMLi group. Therefore, achieving the recommended $100-150 \mathrm{mg} / \mathrm{d}^{(57,58)}$ for children 6-24 months of age, compared with those in the CM (control) group who received $<6 \mathrm{mg} / \mathrm{d}$ (DHA, EPA and DPA) from the study milk.

Whether the consumption of GUM displaces energy intake, impacts dietary variety or inadvertently limits the foods offered to children by their parents due to providing a sense of 'nutritional security' remains an area of concern for parents, paediatric medical professionals and advisory groups ${ }^{(48)}$, and remains an area that has received less research attention. Young children's feeding practices vary markedly and are influenced by cultural, social and economic factors, differing advice from healthcare professionals and availability of products $^{(22)}$. Recommendations and parental perceptions for the use of GUM in European countries vary ${ }^{(22)}$, and our understanding as to what drives New Zealand and Australian parents to use GUM is less understood. In a recent position statement, ESPGHAN do not recommend the routine use of GUM in children from 1 to 3 years of age but do acknowledge that these milks can be used as one of several methods to increase intake of micronutrients such as Fe, vitamin D and $n$-3 PUFA and also as a strategy to reduce the total protein intake ${ }^{(19)}$.

In conclusion, the consumption of a reduced protein GUM (GUMLi) fortified with $\mathrm{Fe}$ and vitamin $\mathrm{D}$, for a period of 12 months did not affect dietary patterns compared with consumption of an unfortified CM. GUMLi resulted in improved intake of several key micronutrients, including $\mathrm{Fe}$ and vitamin $\mathrm{D}$, and reduced intake of protein. It is possible that a whole diet, without the use of GUM, would provide sufficient nutrients, but in the case of increased susceptibility to nutrient inadequacy, the use of GUMLi in moderate amounts could be considered as a strategy to improve total nutrient intakes without affecting dietary patterns or displacing other foods in the diet throughout the second year of life.

\section{Acknowledgements}

Trial registration: Australian New Zealand Clinical Trials Registry (https://www.anzctr.org.au) number: ACTRN12614000918628. Date registered 27/08/2014. This trial received an investigatorinitiated grant from Danone Pty Ltd. The funder had no role in data collection, analysis and interpretation of the study.

P. S. W. D., C. R. W. and C. C. G. developed the GUMLi trial. A. L. L., M. M., R. J. H., C. R. W. and P. S. W. D. wrote the study protocol. A. L. L., M. M., T. M. and R. J. H. conducted the study. A. L. L. and C. R. W. wrote the manuscript. Y. J. and R. X. C. conducted the statistical analyses of the data. All authors have read and approved the final manuscript.

C. R. W. has received honoraria for presentations and consultations from Danone, Nutricia, Pfizer and Fonterra. P. S. W. D. has received honoraria for presentations and consultations from Danone, Nurticia, Nestlé, Mead Johnson and Aspen Nutritionals. C. C. G. has received honoraria for consultations from Fonterra. R. J. H. has received honoraria for presentations and consultation from Danone Nutricia, Nestlé and Aspen Nutritionals. None of the remaining authors report a conflict of interest related to the study. A. L. L., T. M., M. M., Y. J. and R. X. C. have no conflicts of interest. The GUMLi trial received an investigator-initiated grant from Danone Nutricia Research. The funder had no role in the study design, data collection, analysis and interpretation of the study. There are no restrictions or delays on the timely publication of the results of the trial.

\section{Supplementary material}

For supplementary material/s referred to in this article, please visit https://doi.org/10.1017/S0007114518003847

\section{References}

1. National Health and Medical Research Council (2012) Infant Feeding Guidelines: Summary. Canberra: National Health and Medical Research Council.

2. Birch L, Savage JS \& Ventura A (2007) Influences on the development of children's eating behaviours: from infancy to adolescence. Can J Diet Pract Res 68, s1-s56. 
3. Northstone K \& Emmett P (2013) The associations between feeding difficulties and behaviours and dietary patterns at 2 years of age: the ALSPAC cohort. Matern Child Nutr 9, 533-542.

4. Hilger J, Goerig T, Weber P, et al. (2015) Micronutrient intake in healthy toddlers: a multinational perspective. Nutrients $\mathbf{7}$, 6938-6955.

5. Webb K, Rutishauser I \& Knezevic N (2008) Foods, nutrients and portions consumed by a sample of Australian children aged 16-24 months. Nutr Diet 65, 56-65.

6. Livingstone M, Robson PJ \& Wallace J (2004) Issues in dietary intake assessment of children and adolescents. Br J Nutr $\mathbf{9 2}$, Suppl. 2, S213-S222.

7. Robinson S, Marriott L, Poole J, et al. (2007) Dietary patterns in infancy: the importance of maternal and family influences on feeding practice. Br J Nutr 98, 1029-1037.

8. Ambrosini GL (2014) Childhood dietary patterns and later obesity: a review of the evidence. Proc Nutr Soc 73, 137-146.

9. Jolliffe I (2002) Principal Component Analysis. Wiley Online Library.

10. Hu FB (2002) Dietary pattern analysis: a new direction in nutritional epidemiology. Curr Opin Lipidol 13, 3-9.

11. Smithers LG, Golley RK, Brazionis L, et al. (2011) Characterizing whole diets of young children from developed countries and the association between diet and health: a systematic review. Nutr Rev 69, 449-467.

12. Smithers LG, Brazionis L, Golley RK, et al. (2012) Associations between dietary patterns at 6 and 15 months of age and sociodemographic factors. Eur J Clin Nutr 66, 658-666.

13. Baird J, Poole J, Robinson S, et al. (2008) Milk feeding and dietary patterns predict weight and fat gains in infancy. Paediatr Perinat Epidemiol 22, 575-586.

14. Harvey NC, Robinson SM, Crozier SR, et al. (2009) Breastfeeding and adherence to infant feeding guidelines do not influence bone mass at age 4 years. Br J Nutr 102, 915-920.

15. Robinson SM, Marriott LD, Crozier SR, et al. (2009) Variations in infant feeding practice are associated with body composition in childhood: a prospective cohort study. J Clin Endocrinol Metab 94, 2799-2805.

16. Kiefte-de Jong JC, de Vries JH, Bleeker SE, et al. (2013) Sociodemographic and lifestyle determinants of 'Western-like' and 'Health conscious' dietary patterns in toddlers. Br J Nutr 109, 137-147.

17. Smithers LG, Golley RK, Brazionis L, et al. (2012) Dietary patterns of infants and toddlers are associated with nutrient intakes. Nutrients 4, 935-948.

18. Shin KO, Oh S \& Park HS (2007) Empirically derived major dietary patterns and their associations with overweight in Korean preschool children. Br J Nutr 98, 416-421.

19. Hojsak I, Bronsky J, Campoy C, et al. (2018) Young child formula: a position paper by the ESPGHAN committee on nutrition. J Pediatr Gastroenterol Nutr 66, 177-185.

20. Przyrembel H \& Agostoni C (2013) Growing-up milk: a necessity or marketing? World Rev Nutr Diet 108, 49-55.

21. European Commission, Directorate-General for Health and Food Safety (2016) Young Child Formulae: Background Information. Brussels: European Commission.

22. European Commission, Directorate-General for Health and Food Safety (2016) Report from the Commission to the European Parliament and the Council on Young Child Formulae. Brussels: European Commission.

23. Bell LK, Golley RK, Daniels L, et al. (2013) Dietary patterns of Australian children aged 14 and 24 months, and associations with socio-demographic factors and adiposity. Eur J Clin Nutr 67, 638-645.

24. Wall CR, Hill RJ, Lovell AL, et al. (2019) A multi-center, double blind, randomised, placebo controlled trial to evaluate the effect of consuming Growing Up Milk 'lite' on body composition in children aged 12-23 months. Am J Clin Nutr (In the Press).

25. Watson EO, Heath AM, Taylor RW, et al. (2015) Relative validity and reproducibility of an FFQ to determine nutrient intakes of New Zealand toddlers aged 12-24 months. Public Health Nutr 18, 3265-3271.

26. Mills VC, Skidmore PM, Watson EO, et al. (2015) Relative validity and reproducibility of a food frequency questionnaire for identifying the dietary patterns of toddlers in New Zealand. J Acad Nutr Diet 115, 551-558.

27. EFSA Panel on Dietetic Products, Nutrition and Allergies (NDA) (2013) Scientific opinion on nutrient requirements and dietary intakes of infants and young children in the European Union. EFSA J 11, 3408.

28. Taylor BJ, Heath AM, Galland BC, et al. (2011) Prevention of overweight in infancy (POI. nz) study: a randomised controlled trial of sleep, food and activity interventions for preventing overweight from birth. BMC Public Health 11, 942.

29. Huang TT, Roberts SB, Howarth NC, et al. (2005) Effect of screening out implausible energy intake reports on relationships between diet and BMI. Obes Res 13, 1205-1217.

30. Cattell RB (1966) The scree test for the number of factors. Multivariate Behav Res 1, 245-276.

31. Gorsuch RL (1974) Factor Analysis. Philadelphia, PA: WB Saunders.

32. Twisk J, Kemper H \& Mellenbergh GJ (1994) Mathematical and analytical aspects of tracking. Epidemiol Rev 16, 165-183.

33. Luque V, Escribano J, Closa-Monasterolo R, et al. (2018) Unhealthy dietary patterns established in infancy track to midchildhood: the EU childhood obesity project. J Nutr $\mathbf{1 4 8}$, 752-759.

34. National Health and Medical Research Council (2006) Nutrient Reference Values for Australia and New Zealand Including Recommended Dietary Intakes. Canberra: National Health and Medical Research Council.

35. Carriquiry AL (1999) Assessing the prevalence of nutrient inadequacy. Public Health Nutr 2, 23-34.

36. Food and Nutrition Board, Institute of Medicine (2000) Dietary Reference Intakes: Applications in Dietary Assessment. Washington, DC: Institute of Medicine.

37. Ystrom E, Niegel S \& Vollrath ME (2009) The impact of maternal negative affectivity on dietary patterns of 18-monthold children in the Norwegian Mother and Child Cohort Study. Matern Child Nutr 5, 234-242.

38. Lioret S, Betoko A, Forhan A, et al. (2015) Dietary patterns track from infancy to preschool age: cross-sectional and longitudinal perspectives. J Nutr 145, 775-782.

39. Wall CR, Thompson J, Robinson E, et al. (2013) Dietary patterns of children at 3.5 and 7 years of age: a New Zealand birth cohort study. Acta Paediatr 102, 137-142.

40. Bocquet A, Bresson JL, Briend A, et al. (2003) Alimentation du nourrisson et de l'enfant en bas âge. Réalisation pratique (Feeding infants and toddlers. Practical realisation). Arch Pediatr 10, 76-81.

41. Ghisolfi J, Fantino M, Turck D, et al. (2013) Nutrient intakes of children aged 1-2 years as a function of milk consumption, cows' milk or growing-up milk. Public Health Nutr 16, 524-534.

42. Bramhagen AC \& Axelsson I (1999) Iron status of children in southern Sweden: effects of cow's milk and follow-on formula. Acta Paediatr 88, 1333-1337.

43. Walton J \& Flynn A (2013) Nutritional adequacy of diets containing growing up milks or unfortified cow's milk in Irish children (aged 12-24 months). Food Nutr Res 57, 21836.

44. Gibson S \& Sidnell A (2014) Nutrient adequacy and imbalance among young children aged 1-3 years in the UK. Nutr Bull 39, $172-180$. 
45. Soh P, Ferguson EL, McKenzie JE, et al. (2002) Dietary intakes of 6-24-month-old urban South Island New Zealand children in relation to biochemical iron status. Public Health Nutr 5 339-346.

46. Virtanen MA, Svahn C, Viinikka LU, et al. (2001) Iron-fortified and unfortified cow's milk: effects on iron intakes and iron status in young children. Acta Paediatr 90, 724-731.

47. Sazawal S, Dhingra U, Dhingra P, et al. (2010) Micronutrient fortified milk improves iron status, anemia and growth among children 1-4 years: a double masked, randomized, controlled trial. PLOS ONE 5, e12167.

48. Szymlek-Gay EA, Ferguson EL, Heath AM, et al. (2009) Foodbased strategies improve iron status in toddlers: a randomized controlled trial. Am J Clin Nutr 90, 1541-1551.

49. Atkins LA, McNaughton SA, Campbell KJ, et al. (2016) Iron intakes of Australian infants and toddlers: findings from the Melbourne infant feeding, activity and nutrition trial (InFANT) program. Br J Nutr 115, 285-293.

50. Hower J, Knoll A, Ritzenthaler KL, et al. (2013) Vitamin D fortification of growing up milk prevents decrease of serum 25-hydroxyvitamin D concentrations during winter: a clinical intervention study in Germany. Eur $J$ Pediatr 172, 1597-1605.

51. Houghton LA, Gray AR, Szymlek-Gay EA, et al. (2011) Vitamin D-fortified milk achieves the targeted serum 25hydroxyvitamin $\mathrm{D}$ concentration without affecting that of parathyroid hormone in New Zealand toddlers. J Nutr 141, 1840-1846.
52. Food Standards Australia New Zealand (2012) Australia New Zealand Food Standards Code - Standard 1.3.2. Vitamins and Minerals. Canberra: FSANZ.

53. Akkermans MD, Eussen SR, van der Horst-Graat JM, et al. (2017) A micronutrient-fortified young-child formula improves the iron and vitamin D status of healthy young European children: a randomized, double-blind controlled trial. $A m$ J Clin Nutr 105, 391-399.

54. Calvert C, Cade J, Barrett JH, et al. (1997) Using cross-check questions to address the problem of mis-reporting of specific food groups on Food Frequency Questionnaires. UKWCS Steering Group. United Kingdom Women's Cohort Study Steering Group. Eur J Clin Nutr 51, 708-712.

55. Movassagh EZ, Baxter-Jones AD, Kontulainen S, et al. (2017) Tracking dietary patterns over 20 years from childhood through adolescence into young adulthood: the Saskatchewan Pediatric Bone Mineral Accrual Study. Nutrients 9, 990.

56. Tucker KL (2010) Dietary patterns, approaches, and multicultural perspective. Appl Physiol Nutr Metab 35, 211-218.

57. EFSA Panel on Dietetic Products, Nutrition, and Allergies (NDA) (2010) Scientific opinion on dietary reference values for fats, including saturated fatty acids, polyunsaturated fatty acids, monounsaturated fatty acids, trans fatty acids, and cholesterol. EFSA J 8, 1461-1568.

58. FAO, WHO (2010) Expert Consultation on Fats and Fatty Acids in Human Nutrition: Fats and Fatty Acids in Human Nutrition: Report of an Expert Consultation, 10-14 November 2008. Food and Agriculture Organization of the United Nation. 